\title{
A Method of Accurate License Plate Location
}

\author{
Chunli Lin ${ }^{1, a^{*}}$, Yufan $\mathrm{Wu}^{1, \mathrm{~b}}$ and Yajun $\mathrm{Wu}^{1, \mathrm{c}}$ \\ ${ }^{1}$ University of Science and Technology Liaoning, Anshan Liaoning, 114051 ,China \\ aaslc12000@sina.com, b1036952943@qq.com, '356408163@qq.com
}

Keywords: edge detection; mathematical morphology; license plate location;

Abstract. In order to get accurate license plate location, a method of license plate location based on Sobel operator and mathematical morphology is proposed. Sobel operator and Dilation and closing operation are used to obtain approximate position of the license plate. Then three steps are adopted to get accurate license plate location. Experimental results show this method has high accuracy ratio and the good practical value.

\section{Introduction}

License Plate Recognition (LPR) plays an important role in intelligent transportation system. And the license plate location is the critical technique in the LPR. There are some methods of vehicle license plate location:(1) The method of color feature of license plate location[1][2].But its recognition ratio depends on the illumination. (2) The method of texture feature of license plate location. This algorithm has changed the illumination dependence of the color feature, but the false recognition ratio is high for the complex situation of license plate image [3].(3) License plate location method based on Neural Network[4][5][6].But its calculation is too large and complex in the training phase. In this paper, a new vehicle license plate location method based on Sobel operator and mathematical morphology is proposed, which has high accuracy rate and the good practical value.

\section{License plate preprocessing}

Image preprocessing is an essential process in license plate location and recognition system. It directly affects the recognition ratio.

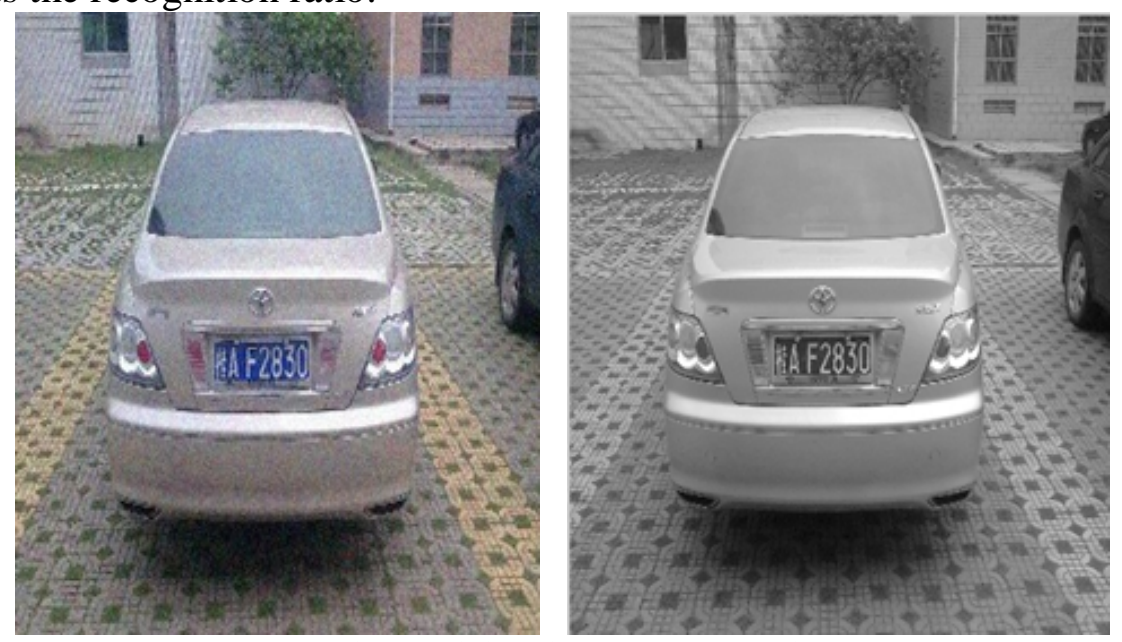

Fig.1 Original image and Gray image

Edge detection: Edge often occurs between the object and the background, or between two objects . In the process of the license plate recognition ,the purpose of the edge detection is to get the license plate information. The gradation among license plate character color, background color and border color changes fast, it is easy to produce significant edge information. Generally there are some edge detection operators, such as Robert operator, Sobel operator and Prewitt operator.

Sobel edge detection operator: The operator can be obtained by convolving the original image $f(x, y)$ with convolution kernel ${ }^{g_{1}}(x, y)$, convolution kernel $g_{2}(x, y)$.Its mathematical expression is shown in (1). 


$$
S(x, y)=\operatorname{MAX}\left[\sum_{m=1}^{M} \sum_{n=1}^{N} f(m, n) g_{1}(i-m, j-n), \sum_{m=1}^{M} \sum_{n=1}^{N} f(m, n) g_{2}(i-m, j-n)\right]
$$

Sobel operator, just as the same as Prewitt operator, computes an approximation of the gradient of an image intensity function. In general, it is the kernel size of 3.Horizontal change is computed by convolving $f(x, y)$ with a kernel $g_{1}(x, y), g_{1}(x, y)$ would be computed as:

$$
\left(\begin{array}{ccc}
-1 & 0 & -1 \\
-2 & 0 & 2 \\
-1 & 0 & 1
\end{array}\right)
$$

Vertical change is computed by convolving $f(x, y)$ with a kernel $g_{2}(x, y), g_{2}(x, y)$ would be computed as:

$$
\left(\begin{array}{ccc}
-1 & -2 & -1 \\
0 & 0 & 0 \\
1 & 2 & 1
\end{array}\right)
$$

The Sobel Operator combines Gaussian smoothing and differentiation, so it can detect the edge points and remove the noise .Its mathematical expression is shown in (2)-(4)

$$
\begin{aligned}
& S_{1}(i, j)= f(i-1, j-1)+2 f(i-1, j)+f(i-1, j+1) \\
&-f(i+1, j-1)-2 f(i+1, j)-f(i+1, j+1) \\
& S_{2}(i, j)= f(i-1, j+1)+2 f(i, j+1)+f(i+1, j+1)- \\
& f(i-1, j-1)-2 f(i, j-1)-f(i+1, j-1) \\
& S(i, j)=\left|S_{1}(i, j)\right|+\left|S_{2}(i, j)\right|
\end{aligned}
$$

In order to satisfy the real-time requirements, Sobel operator has advantages of high accuracy and fast processing speed,so it was taken to detect the edge. The edge of the image is shown in Fig. 2.

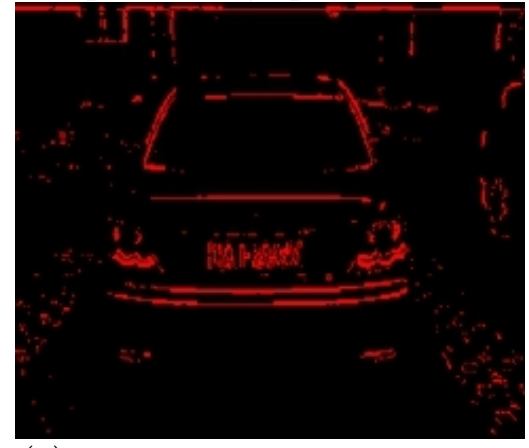

(a) Edge of Roberts operator

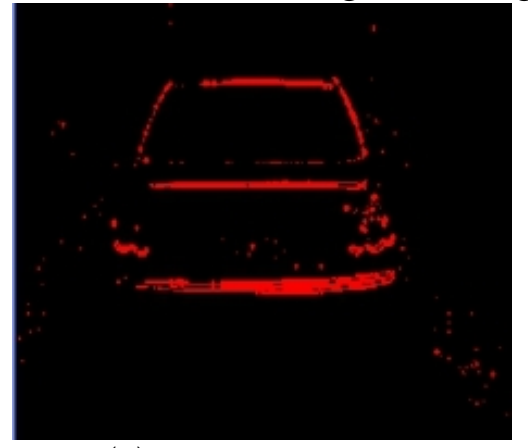

(b) Edge of Prewitt operator

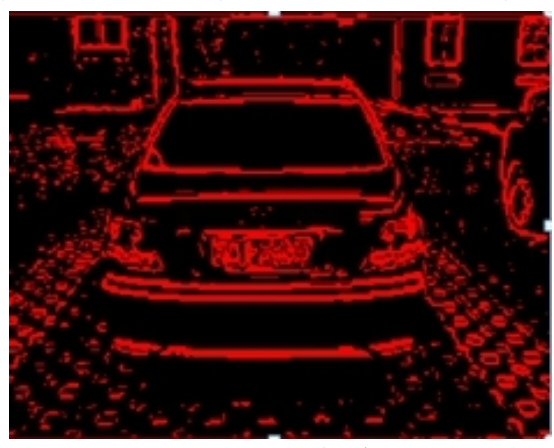

(c) Edge of Sobel operator

Fig.2 Image contrast after process by several edge detection operator

\section{Approximate location by mathematical morphology}

From Fig. 2, we can see that the license plate area in the background can be clearly displayed. But there are still many edges, such as a automobile contour and the characters edge on the label of the car. Now, Connected domain of mathematical morphology is used to remove interference (the other edges).

Mathematical morphology (MM) is a theory and technique for the analysis and processing of geometrical structures. It mainly extracts useful components from a image, such as skeleton, edge.

The basic morphological operators are erosion, dilation, opening and closing [7].

Let $E$ be a Euclidean space or an integer grid, and a binary image in $E$.

Erosion: The erosion of the binary image $A$ by the structuring element $B$ is defined by: 


$$
A \Theta B=\left\{(x, y) \in E \mid(B)_{(x, y)} \subseteq A \neq \varnothing\right]
$$

where $\mathrm{B}(\mathrm{x}, \mathrm{y})$ is the translation of $B$ by the vector $(\mathrm{x}, \mathrm{y})$.

Dilation: The dilation of $\mathrm{A}$ by the structuring element $\mathrm{B}$ is defined by:

$$
A \oplus B=\bigcup_{B=B} A_{s}
$$

The dilation is commutative, also given by:

$$
A \oplus B=B \oplus A=\bigcup_{a \in A} B_{a}
$$

Dilation and erosion are not reversible operation. They can be used successively to be "opening" and "closing".

Opening:The opening of $A$ by $B$ is obtained by the erosion of $A$ by $B$, followed by dilation of the resulting image by $B$ :

$$
A \circ B=(A \oplus B) \oplus B
$$

Closing: The closing of $A$ by $B$ is obtained by the dilation of $A$ by $B$, followed by erosion of the resulting structure by $B$ :

$$
A \bullet B=(A \oplus B) \oplus B
$$

The dilation has the effect of enlarging the target object pixels. Erosion has the effect of shrinking target pixels. The number of pixels are determined by the size and shape of the structure elements. Because the license plate area is a fixed rectangle, first, we can choose the dilation of A by the structuring element B of $2 * 6$. Then a closing operation is adopted to eliminate some of the small gap in the connected domain. The result is shown in Fig. 3. The approximate position of the license plate can be seen in Fig.3.

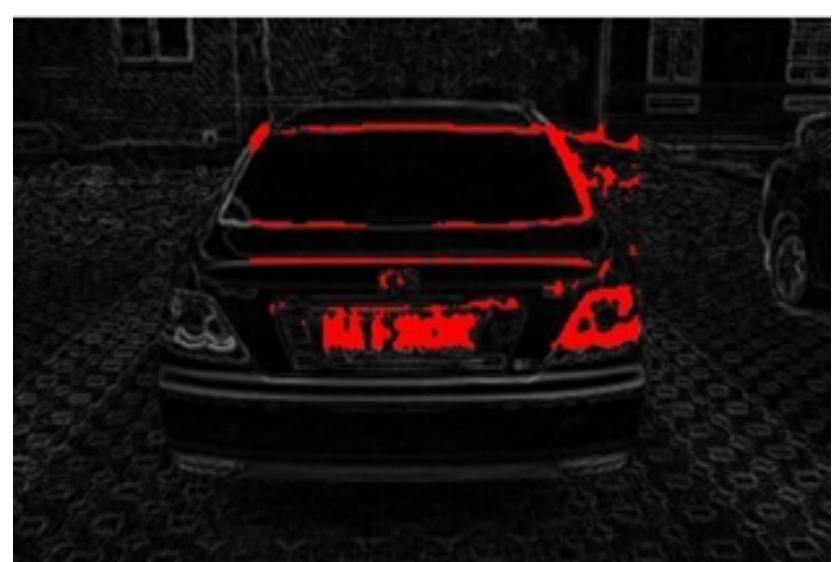

Fig. 3 image after dilation and closing operation

\section{Accurate license plate location}

Through the analysis of the edge and mathematical morphology, we obtain some connected domains, but only one is needed, which is the real license plate region.

According to the inherent characteristics of the license plate region, some factors are analyzed for each candidate region.

Step 1. Calculate the ratio of length to width of every candidate region. Only the ratio is in $[2,5]$, the candidate region will be retained.

Step 2.Calculate the area of the retained region. If the area is in $[2500,7500]$, The corresponding region will be kept.

Step 3.Check color component in the region. If it meets the color saturation of the license plate in China, it will be the region we look for.

Accurate position of the license plate region is obtained by the minimum enclosing rectangle method, shown in Fig.4. 


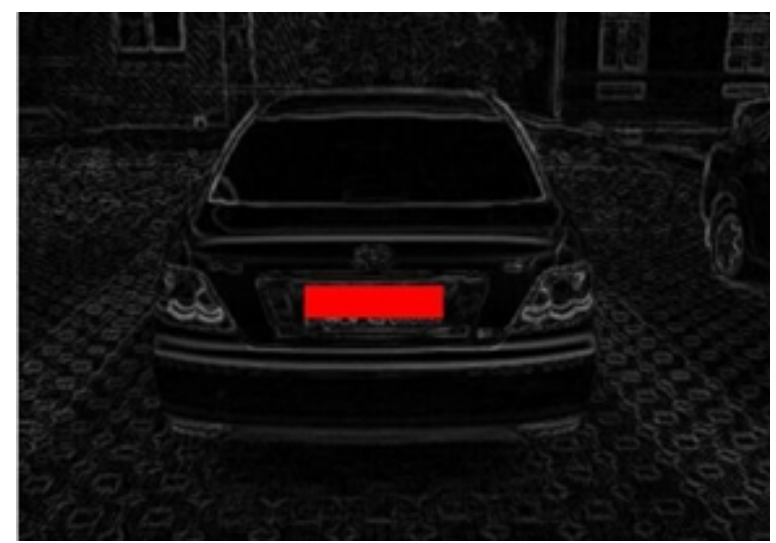

Fig. 4 Connected domain of plate

\section{Display location results}

After that, accurate position of the license plate region is found. The contents of the red rectangle part of the license plate region recorded in Fig.4 is extracted, shown in Fig.5.

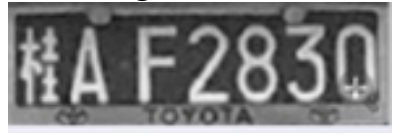

Fig. 5 Region of license plate

\section{Summary}

In this paper, an efficient vehicle license plate locating method called combination of sobel operator and mathematical morphology is proposed. Then three steps are adopted to get accurate license plate location. Experiment steps are given in detail. Experimental results show that the algorithm is feasible. The obtained recognition ratio is about $98 \%$ for a 65 random images included in the database. The algorithm only failed in a slanted license plate.

\section{References}

[1] Wang H, Wang X, Li W, et al. Color prior knowledge-based license plate location algorithm [C] Digital Media and its Application in Museum \& Heritages, Second Workshop on. IEEE (2007)47-52.

[2] Luo Z, Cao J. Vehicle license plate location algorithm based on gray level variation and color features[C] Image and Signal Processing, 2009. CISP' 09. 2nd International Congress on. IEEE (2009) $1-4$.

[3] Xiao-yan, Yao-quan, Wen-ting. Image Edge Detection Algorithms and Their Application in P ower Plants[J]. Information On Electric Power, 2002.

[4] Li Yucheng, Yang Guangming, Wang Mushu. Study and Design for System of License Plate Extraction and Recognition, J. Computer Measurement \& Control. 19(1) (2011).158-160.

[5] ZHANG Yan, REN An-hu. Research on Multiple Feature and BP Neural Network License Plate Recognition System. J. Science Technology and Engineering. 12(22)(2012).5645-5648.

[6] CHEN Jian-kun, FAN Chun-nian. Neural network based vehicle license plate location, J. Journal of Liaoning Technical University, J. 24(1)(2005).97-100.

[7] Information on https://en.wikipedia.org/wiki/Mathematical_morphology 